\title{
Investigation of young children with severe recurrent wheeze: any clinical benefit?
}

\author{
S. Saglani*, A.G. Nicholson\#, M. Scallan ", I. Balfour-Lynn*, M. Rosenthal*, \\ D.N. Payne* and A. Bush*
}

ABSTRACT: The management of young children with severe recurrent wheeze is difficult because symptoms are often refractory to conventional asthma therapy and other diagnoses must be excluded. The present authors aimed to evaluate the outcome of detailed, invasive investigations in such patients.

Children aged between 3 months and 5 yrs with severe recurrent wheezing, who had been referred to a tertiary centre, underwent a protocol of investigations including a chest computed tomography scan, blood tests, nasal ciliary brushings, fibreoptic bronchoscopy, bronchoalveolar lavage (BAL), endobronchial biopsy and passage of an oesophageal $\mathrm{pH}$ probe.

A total of 47 children (25 males) with a median age of 26 (range 5-58) months underwent investigation. Of these, $39 \%$ were atopic, two-thirds had evidence of gastro-oesophageal reflux and 37 out of 47 had an abnormal bronchoscopy. Findings included structural abnormalities (13 out of 37 ), excessive mucus (20 out of 37 ) and macroscopic inflammation (10 out of 37 ). BAL revealed bacterial growth in 12 out of 44 (27\%) patients. Good quality endobronchial biopsies were obtained from 36 out of $46(78 \%)$ patients; of these, $44 \%$ had tissue eosinophila and $28 \%$ had a thickened reticular basement membrane.

Additional investigations (including bronchoscopy) in young children with severe wheeze may help to identify positive diagnoses and provide information to support a clinical diagnosis of asthma. This hypothesis-generating work should form the basis of future interventional studies.

KEYWORDS: Bronchoscopy, endobronchial biopsy, investigations, paediatric, preschool, wheeze

W heezing in infants and preschool children is common [1] and accounts for many acute hospital admissions [2]. The management of these patients, especially when symptoms are recurrent and severe, is a challenging task because of many difficult issues unique to this age group [3]. Current practice tends to focus on confirming the presence of wheeze [4] (and distinguishing it from stridor and other upper airway noises) and then prescribing therapy targeted at the most likely cause. Excluding diagnoses other than asthma and gathering information that supports a diagnosis of asthma are probably the most important but also the most difficult issues to address in this age group [5]. Prescribing therapy targeted at the most likely cause of wheezing is therefore the most common approach in managing these patients, often resulting in therapeutic trials of asthma treatment, such as bronchodilators, inhaled corticosteroids and montelukast [6]. However, in a proportion of patients with recurrent wheeze, symptoms are refractory to conventional asthma therapies [7] and the safety and efficacy of more unusual treatments has not been tested [8], resulting in referral to a tertiary centre for further assessment. Furthermore, there have been very few attempts to delineate the underlying disease in this difficult group of patients.

In the unit of the present authors, the evaluation of such patients involves a series of investigations aimed at making a positive diagnosis and then treating accordingly. Investigation involves specific tests to identify conditions other than asthma, such as gastro-oesophageal reflux (GOR), primary ciliary dyskinesia, obliterative bronchiolitis, bronchiectasis, structural airway abnormalities, lower respiratory tract infections and immunodeficiency. Investigations are also performed to obtain information that may support a diagnosis of asthma. These include tests to confirm atopy [9], such as serum immunoglobulin (Ig)E and the radioallergosorbent test (RAST), assessment of the extent of eosinophilic airway inflammation in bronchoalveolar lavage (BAL) and endobronchial biopsy, and
AFFILIATIONS

*Depts of Respiratory Paediatrics,

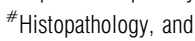

"Anaesthetics, Royal Brompton

Hospital, London, UK.

CORRESPONDENCE

A. Bush

Dept of Respiratory Paediatrics Royal Brompton Hospital

Sydney Street

London SW3 6NP

UK

Fax: 442073518763

E-mail: a.bush@rbh.nthames.nhs.uk

Received

March 152005

Accepted after revision:

September 172005
European Respiratory Journa Print ISSN 0903-1936 Online ISSN 1399-3003 
measurement of reticular basement membrane (RBM) thickness in biopsies. This approach is intended to allow subsequent management to be rationalised, with treatment targeted at specific conditions.

This is a retrospective review of the usefulness of an aggressive and thorough clinical approach to investigating these children. The present authors aimed to assess the clinical gain from performing the outlined set of investigations in preschool children with severe recurrent wheeze who were referred to a tertiary respiratory centre for a further opinion. The intention was for these data to be hypothesis generating, and serve as a basis for future interventional studies.

\section{METHODS \\ Subjects}

All infants and young children aged between 3 months and 5 yrs referred to the Royal Brompton Hospital (London, UK) for further investigation of severe recurrent wheeze between November 2002 and December 2004 were eligible for inclusion. After assessment in the out-patient clinic by a consultant respiratory paediatrician, a decision was made whether to perform additional investigations. This was discussed with the family, who were offered the choice between continued empirical manipulation of medical therapy, or detailed investigation to try to establish a targeted treatment plan. They were informed of the possibility that investigation might not lead to a new diagnosis. All investigations were carried out with full informed parental consent and included a written information sheet giving full details about the bronchoscopy, BAL and biopsy (available from the authors on request).

\section{Inclusion criteria}

All patients had recurrent, severe, noisy breathing, which had been interpreted as wheeze, either when assessed during acute symptoms or from parental reports. They all had at least three episodes of wheeze in the previous 6 months. A total of 11 out of $47(23 \%)$ had symptoms precipitated only by colds. Although previous hospital admission with wheeze was not an inclusion criterion, 41 out of 47 (87\%) patients had been admitted at least once with acute wheeze. All patients had already had a failed trial of bronchodilator therapy. All had a previous trial of inhaled or oral steroid therapy. The minimum duration of the trial of inhaled steroids was 2 months. Out of 47 patients, 28 were prescribed inhaled steroid therapy at the time of investigation. All patients remained symptomatic despite therapy. Eight patients were born between 33 and 36 weeks gestation, but were all an appropriate weight for gestational age at birth and had required no respiratory support after birth. Three patients were born at term but were small for gestational age [10].

\section{Exclusion criteria}

Patients with isolated cough without associated noisy breathing were not included, neither were those whose main problem was recurrent lower respiratory tract infection. Patients who had required oxygen or ventilation in the neonatal period and those who were currently oxygen dependent were excluded.

\section{Investigations}

A defined group of tests were arranged for all patients. If some tests had already been performed at the local hospital, they were not always repeated. The investigations were performed during an overnight stay in hospital, the majority being carried out under general anaesthesia, as this is the method used to perform bronchoscopy at the institution of the current authors. The investigations are summarised in table 1.

\section{Blood tests}

Total IgE and RASTs to milk, egg, peanut, house dust mite, cat, dog, grass and tree pollens, and $\operatorname{IgG}, \operatorname{Ig} A, \operatorname{IgM}$ and $\operatorname{IgG}$ subclasses were measured.

\section{Sweat test}

Sweat tests were performed using quantitative pilocarpine iontophoresis, according to national guidelines [11].

\section{High-resolution CT chest scan}

All high-resolution computed tomography (HRCT) chest scans were obtained during quiet tidal breathing. Sections were acquired at $10-\mathrm{mm}$ intervals in the supine position using an electron beam ultrafast scanner (Imatron Inc.; San Francisco, CA, USA). Scans were reported by one of three consultant radiologists.

\section{Oesophageal $\mathrm{pH}$ monitoring}

A multi-use, single-channel $\mathrm{pH}$ catheter was passed whilst the patient was still anaesthetised at the end of the bronchoscopic examination. Continuous $\mathrm{pH}$ monitoring was performed using a Synectics Digitrapper Mark III (Synectics Inc.; Irving TX, USA). The position of the catheter was checked on a chest radiograph performed immediately after the procedure and adjusted if necessary so that the tip of the catheter was between the 8 th and 10th thoracic vertebrae. An oesophageal $\mathrm{pH}$ of $<4$ for $>4 \%$ of recording time (minimum duration of $18 \mathrm{~h}$ ) was considered abnormal, except in infants, when age-appropriate values were used [12].

\section{Nasal ciliary brushings}

Samples were taken under general anaesthetic and analysed as previously described [13].

\begin{tabular}{ll} 
TABLE 1 & $\begin{array}{l}\text { Investigations performed during an overnight } \\
\text { stay in young children with severe recurrent } \\
\text { Wheeze }\end{array}$ \\
& Investigation \\
\hline Day 1 & Sweat test \\
Under oral sedation, if needed & High-resolution CT chest scan \\
Under general anaesthetic & Blood tests: total IgE, RASTs to food \\
& and aeroallergens, IgG, IgA, IgM \\
& and lgG subclasses \\
& Nasal ciliary brushings \\
& Fibreoptic bronchoscopy with \\
& bronchoalveolar lavage and \\
& endobronchial biopsies \\
& Placement of oesophageal pH probe \\
CT: computed tomography; Ig: immunoglobulin; RAST: radioallergosorbent \\
test.
\end{tabular}




\section{Bronchoscopy, BAL and endobronchial biopsies}

Fibreoptic bronchoscopy was performed under general anaesthetic, by one of three clinicians, as previously described [14]. A flexible bronchoscope (size $2.8 \mathrm{~mm}$; Olympus, KeyMed; Southend-on-Sea, UK) was used for children aged $<2 \mathrm{yrs}$, and a 3.6-mm scope (Olympus, KeyMed) was used for those aged $>2$ yrs. Both bronchoscopes have the same size $(1.2 \mathrm{~mm})$ instrument channel. Endobronchial biopsies were taken using appropriately sized rat tooth biopsy forceps (serial number FB56D-1; Olympus, Keymed). The fibreoptic bronchoscope was passed through a facemask into the nostril, while anaesthesia was maintained by sevofluorane. The upper and lower airways were inspected for evidence of malacia (assessed during quiet spontaneous breathing) or anatomical abnormalities, such as enlarged adenoids and tonsils, and for the presence of excess mucus, macroscopic inflammation and oedema. BAL was performed in the right middle or lower lobe using three aliquots of $1 \mathrm{~mL} \cdot \mathrm{kg}^{-1}$ of normal saline [15]. BAL fluid was processed for bacterial culture and viral immunofluorescence. It was also evaluated qualitatively for the presence of fat-laden macrophages and inflammatory cells. The results were reported as follows: 1) fat-laden macrophages: none, small, moderate or large (any reported fat-laden macrophages were considered abnormal); and 2) inflammation: none, eosinophilia, neutrophilia or combined eosinophilia and neutrophilia. Up to four endobronchial biopsies were taken from the subcarinae of the right lower lobe. Biopsies were fixed and processed into paraffin blocks. Step sections $(5-\mu \mathrm{m}$ thick) were cut $50 \mu \mathrm{m}$ apart and stained with haematoxylin and eosin. Inflammation and structural changes in biopsies were scored semiquantitatively as follows: goblet cell hyperplasia (0-6); basement membrane thickening (0-3); lymphocytes/plasma cells (0-6); neutrophils (0-6); eosinophils (0-6); and seromucinous gland hyperplasia (0-3). Based on control data from biopsies taken from age-matched patients with stridor, the present authors defined RBM thickening as a score $>1$, and tissue eosinophilia was indicated by a score $>0$.

\section{Analysis of data}

The data was initially analysed for the group as a whole, and further analysed with the patients divided into three groups according to age, with Group 1: $<18$ months; Group 2: 18-36 months; and Group 3: $>36$ months. Nonparametric tests were applied to test for intergroup differences of numerical variables. Comparison between all groups was made using the Kruskal-Wallis test, followed by a Mann-Whitney U-test if a significant difference $(p<0.05)$ was found. The Bonferroni correction was introduced for multiple comparisons. The Chisquared test was used for categorical variables.

\section{RESULTS}

Patient details are summarised in table 2. A total of 28 out of 47 $(60 \%)$ were currently prescribed inhaled corticosteroids, eight out of $47(17 \%)$ were taking a short course of oral steroids and three out of $47(6 \%)$ were using long-term oral steroids at the time of investigations. The number of patients that had each test and the results are summarised in table 3 . Of note, there were no abnormal cilia or sweat test results. Total IgE was measured in 46 out of 47 subjects with a median value of 23 (range $1-2,604) \mathrm{IU} \cdot \mathrm{mL}^{-1}$. Only two patients had an $\operatorname{IgE}>2 \mathrm{SD}$ above normal [16] for their age. In total, 46 out of 47 patients

\begin{tabular}{ll} 
TABLE 2 & $\begin{array}{l}\text { Clinical characteristics of patients, illustrating risk } \\
\text { factors for asthma and wheeze severity }\end{array}$ \\
\hline Patients $\mathbf{n}$ & 47 \\
Age months & $26(5-58)$ \\
Sex males & $25(53)$ \\
Hospital admissions for acute wheeze & $41(87)$ \\
Number of admissions per patient & \# \\
Eczema & $3.5(1->10)$ \\
Parental asthma & $22(47)$ \\
Symptoms only with colds & $18(38)$ \\
Patients on inhaled steroids at time of & $11(23)$ \\
$\quad$ investigations & $28(60)$ \\
Daily dose of inhaled steroids (budesonide & $400(100-2000)$ \\
$\quad$ equivalent) $\boldsymbol{\mu g}$ &
\end{tabular}

Data are presented as median (range) or $\mathrm{n}(\%)$, unless otherwise stated. ${ }^{\#}$ Actual number of admissions not recorded if $>10$.

had RAST tests performed, of whom 18 (39\%) had at least one positive test.

\section{HRCT scans}

Abnormalities were found on 15 HRCT scans. Three had a structural abnormality (a narrowed left main bronchus $(n=2)$ and an unexpected foreign body that had eroded into the trachea from the oesophagus $(\mathrm{n}=1))$. Six had evidence of small airways disease or air trapping, four had bronchial wall thickening and two had bronchiectasis.

\section{Gastro-oesophageal reflux}

The median percentage of time with a $\mathrm{pH}$ of $<4$ in the $\mathrm{pH}$ study was 8.7 (range $1-25.8) \%$. The result was abnormal in 25 out of $37(68 \%)$ patients. Twenty-one of these (57\%) had an abnormal $\mathrm{pH}$ study and fat-laden macrophages in BAL.

\section{Bronchoscopy, BAL and endobronchial biopsy}

Bronchoscopy was performed in all 47 patients; of these, 37 had abnormal bronchoscopic findings. A total of 13 out of 37 $(36 \%)$ had a structural abnormality (including enlarged tonsils and adenoids causing dynamic airway obstruction during quiet breathing (not previously appreciated during multiple physical examinations), laryngo-, tracheo- or bronchomalacia, foreign body or external, pulsatile tracheal compression). Increased mucus was present in 20 out of $37(54 \%)$ patients, and macroscopic inflammation in 10 out of $37(27 \%)$ patients. BAL was performed in 44 patients and assessed for inflammatory cell profile in 37 out of 44 samples (table 3). Positive bacterial growth was obtained in 12 out of $44(27 \%)$ patients; organisms identified included Branhamella catarrhalis $(\mathrm{n}=3)$, Haemophilus influenzae $(\mathrm{n}=5)$, Streptococcus pneumoniae $(\mathrm{n}=1)$, both Branhamella catarrhalis and Haemophilus influenzae $(\mathrm{n}=2)$, and both methicillin-resistant Staphylococcus aureus and Haemophilus influenzae $(\mathrm{n}=1)$. Viruses were not detected either by immunofluorescence or culture from any BAL sample.

One patient did not have a biopsy due to the presence of a tracheal foreign body. Of the remaining 46 patients, 36 out of $46(78 \%)$ had a biopsy of good enough quality for a clinical report. Of the biopsy parameters assessed, eosinophilic 
TABLE 3 Summary of the results of investigations performed

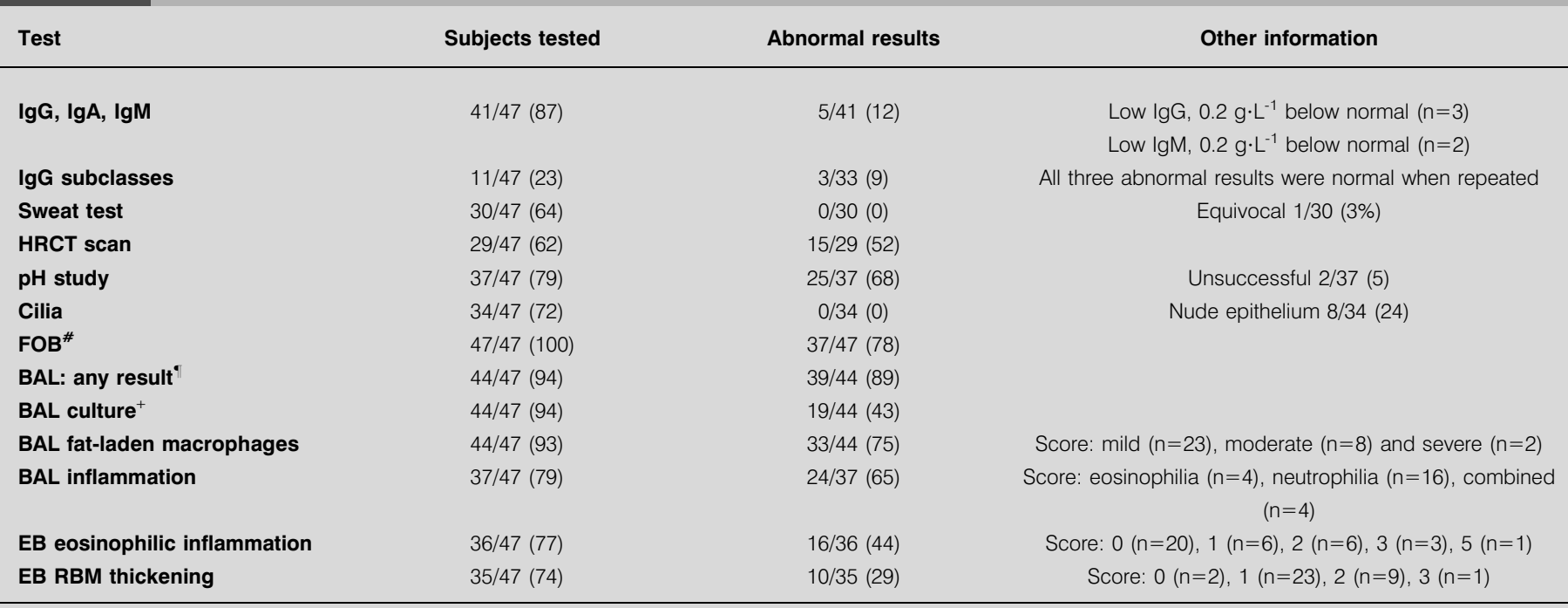

Data are presented as n/n (\%). Ig: immunoglobulin; HRCT: high-resolution computed tomography; FOB: fibreoptic bronchoscopy; BAL: bronchoalveolar lavage; EB endobronchial biopsy; RBM: reticular basement membrane. ${ }^{*}$ : details of abnormalities in the text; ${ }^{\circ}$ : BAL inflammation, fat-laden macrophages or infection; ${ }^{+}$: BAL with significant bacterial growth

inflammation and RBM thickening were the two features of most interest in this group of patients. In total, $44 \%$ had evidence of biopsy eosinophilia and $28 \%$ had RBM thickening. Of the 36 patients, six (17\%) had both abnormalities; five of these were on inhaled steroids and one was on oral steroids.

The clinical interpretation of the results for each patient is presented as diagnostic categories in table 4 . The investigations revealed a potentially clinically meaningful abnormality in $36 / 47(76 \%)$ cases.

\section{Division of subjects into three groups according to age}

Although total IgE increased with age, the number of atopic patients in each age group was similar. Also, significantly more patients aged $<18$ months had structural airway abnormalities at bronchoscopy (table 5). The number of patients with good quality endobronchial biopsies was similar in each age group, but almost all patients with a significantly thickened RBM were in the eldest age group (table 5).

\section{Relationship between peripheral blood markers of atopy and biopsy eosinophilia}

Patients with tissue eosinophilia (score $>0$ ) had a significantly higher total IgE than those without (median (range) IgE 16 (1635) IU versus 6.0 (1-309) IU; $p<0.01$ ). Moreover, significantly more atopic patients had biopsy eosinophils than nonatopic patients (Chi-squared test with Yates' correction $=10.874$; $\mathrm{p}<0.01)$.

\section{DISCUSSION}

Managing young patients with severe, recurrent wheeze can be difficult, and it may be helpful to make a positive diagnosis in order to guide appropriate therapy. To achieve this, a number of investigations can be performed, some of which are relatively invasive. The main finding of the present study is that such invasive investigation of infants and young children with severe recurrent wheeze, who remain symptomatic despite a trial of inhaled steroids, yields abnormal results in three-quarters of cases. The assessment of the clinical

TABLE 4 Clinical meaning of the results of investigations performed in young children with severe, recurrent wheeze

1. Asthma (presence of EB or BAL eosinophilia and/or thickened RBM)

2. Predominantly GOR (abnormal pH study and BAL fat-laden macrophages and normal biopsy)

3. Predominantly infection (significant bacterial growth and neutrophilia in BAL)

4. No definite diagnosis
19/47 (41) (11/19 also had GOR, defined as an abnormal $\mathrm{pH}$ study and BAL fat-laden macrophages) 11/47 (23) (those with GOR in category 1 not included)

6/47 (13) (a total of 12 patients had evidence of infection, but six of these also had GOR, and are in category 2).

$\begin{array}{ll}28(7-58) & 4 / 19(21) \\ 28(5-46) & 3 / 11(27) \\ 9.5(8-13)^{\#} & 3 / 6(50)\end{array}$

$28(6-57)$ $3 / 11(27)$

Data are presented as median (range) or n/n (\%). UAA: structural upper airway abnormality on bronchoscopy; EB: endobronchial biopsy; BAL: bronchoalveolar lavage;

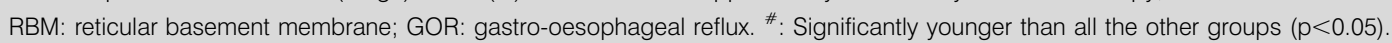


TABLE 5 Results of the investigations in young children with severe wheeze (divided according to age group)

\begin{tabular}{|c|c|c|c|c|}
\hline & \multicolumn{3}{|c|}{ Group } & \multirow[t]{2}{*}{$\mathrm{p}$-value } \\
\hline & $<\mathbf{1 8}$ months & 18-36 months & $>36$ months & \\
\hline Subjects $\mathbf{n}$ & 19 & 13 & 15 & \\
\hline On inhaled steroids & $7 / 19(37)$ & 10/13 (77) & 11/15 (73) & $<0.05$ group 1 versus 2 and 3 \\
\hline On oral steroids & $4 / 19(21)$ & $4 / 13(31)$ & $3 / 15(20)$ & NS \\
\hline CT scan abnormality & 6/11 (55) & 2/9 (22) & $7 / 11(63)$ & NS \\
\hline Abnormal pH study & $7 / 14(50)$ & $8 / 10(80)$ & $10 / 11(91)$ & $<0.05$ group 1 versus 2 and 3 \\
\hline $\begin{array}{l}\text { Structural abnormality on } \\
\text { FOB }\end{array}$ & $10 / 19(53)$ & $1 / 13(8)$ & 2/15 (13) & $<0.01$ group 1 versus 2 and 3 \\
\hline $\begin{array}{l}\text { Positive bacterial growth on } \\
\text { BAL }\end{array}$ & $9 / 16(56)$ & $5 / 13(38)$ & 5/15 (33) & NS \\
\hline Abnormal IgG, IgA, IgM & 2/14 (14) & 0/12 (0) & 3/15 (20) & NS \\
\hline
\end{tabular}

Data are presented as n/n (\%). Ig: immunoglobulin; RAST: radioallergosorbent test; CT: computed tomography; FOB: fibreoptic bronchoscopy; BAL: bronchoalveolar lavage; EB: endobronchial biopsy; ns: nonsignificant.

significance of these results requires prospective intervention trials.

GOR was the predominant finding in most cases. Therefore, it might be reasonable to prescribe an empirical trial of antireflux therapy or perform a $\mathrm{pH}$ study in all preschool children with severe, recurrent wheeze before considering further investigations. However, it is also arguable how many untargeted therapeutic trials are permissible before submitting a child to investigation. Many individuals will have had trials of bronchodilators and inhaled steroids, and repeated blind trials may cause a delay in establishing an important diagnosis, such as upper airway obstruction or an endobronchial foreign body. Furthermore, for patients in whom aspiration is considered likely, bronchoscopy can provide additional information by measuring BAL neutrophils [17] and fat-laden macrophages [18]. It is important to note that the presence of some fat-laden macrophages in the BAL may be completely normal [19] and this is reflected in the present finding of a larger percentage of patients having fat-laden macrophages in BAL than the percentage with an abnormal $\mathrm{pH}$ study. The true significance of fat-laden macrophages in BAL can only be inferred in a prospective, interventional study.

A previous study investigating the prevalence of GOR in preschool children with respiratory symptoms reported that $86 \%$ of patients with GOR did not have gastrointestinal symptoms, and $40 \%$ of those with reactive airways disease had GOR [20]. In another study, $64 \%$ of infants with wheeze were shown to have GOR, two-thirds of which were able to discontinue inhaled steroid therapy within 3 months of starting anti-reflux therapy [21]. However, in older children, even though there appears to be an association between asthma and GOR [22, 23], there is little evidence to suggest that treating GOR results in symptomatic improvement [24, 25].
The role of GOR in contributing to severe wheeze in young children remains uncertain and can only be established with future interventional studies. These reviews have helped to estimate the size of the problem in such patients [22-25].

An important consideration for the current study was how often the performance of bronchoscopy provided useful clinical information (as this procedure is invasive) and involves administration of a general anaesthetic at the present authors' centre. In agreement with the current author's previous data [14], all bronchoscopies were performed without significant complications. Bronchoscopy was abnormal in 37 out of $47(79 \%)$ patients. The detection of macroscopic structural abnormalities in 13 out of $47(28 \%)$ was especially useful. Of note, nine of these patients were between 3 and 18 months old and comprised half of all the patients studied in that age group. This concurs with a previous report of the usefulness of bronchoscopy and BAL in young children with recurrent wheezing, which reported airway abnormalities in 17 out of 30 patients aged between $0-18$ months [26]. In terms of management, if enlarged tonsils and/or adenoids were seen, the relevance of this was investigated further by performance of a sleep study, as the upper airway abnormality may have been contributing to the overall severity of symptoms. The presence of bronchomalacia, without associated airway eosinophilia, identified the cause of wheeze as secondary to a structural airway abnormality, rather than asthma, and encouraged discontinuation of the inhaled steroid therapy. External tracheal compression was further evaluated for the presence of a vascular ring; one patient had an inhaled foreign body.

The presence of a significant bacterial growth, with BAL neutrophilia, was a finding that would suggest the need for appropriately guided initiation of antibiotics. Interestingly, as a 
group, the patients in this predominantly infected diagnostic category were significantly younger than those in the other three categories. This is also in agreement with a previous report in which children with a positive bacterial growth were younger [26].

In terms of histology, the number of patients from whom a good quality endobronchial biopsy (for a clinical report) was obtained was consistent with the current authors' previous findings [14]. The biopsy and BAL cytology report provided useful information on the presence or absence of eosinophilic inflammation. Twelve out of the 16 patients who had eosinophilia on biopsy had been prescribed inhaled steroids, and seven of these 12 were also on oral steroids at the time of bronchoscopy. This may mean that they did not adhere to therapy. However, the presence of airway eosinophilia potentially provides evidence that can help to optimise the dose and improve the delivery of inhaled steroids. This is important as inappropriately high-dose inhaled steroids may have significant side-effects, including severe hypoglycaemia [27], along with possible adverse effects on alveolar development [28].

The findings reported here do not mean that the presence of airway eosinophilia is diagnostic of asthma, even in a wheezing child. The significant association of total $\operatorname{IgE}$ and the presence of one or more positive RAST tests, although suggestive that the airway eosinophilia in the present young children was significant, are not definitive. However, the current authors would suggest that the absence of any evidence of airway eosinophilia should militate against escalating the dose of inhaled corticosteroids.

It was interesting to note that all but one of the patients who had a thickened RBM were $>36$ months old. Little is known about the early onset of structural airway changes in preschool children with severe wheeze, but this concurs with a previous report that showed absence of RBM thickening in wheezy patients, with a median age of 12 months [29].

It should be emphasised that these findings must not be taken as being applicable to all wheezing preschool children. The present authors studied a highly select group, who had severe, recurrent symptoms despite high-dose inhaled steroids and, in some cases, oral steroids. Another limitation of the current study, with respect to the accuracy of the exact proportion represented in each clinical category, was that not all patients underwent all the investigations. Also, the parents of some children who were offered investigations may have opted to continue with empirical therapy. This reflects the retrospective nature of the present study. Patients underwent investigation as part of their clinical assessment rather than according to a strict research protocol. Some had already undergone investigations at their local hospital and in some cases it was felt that certain investigations were not indicated from the clinical picture. However, results from the subgroup of patients who had a HRCT scan, pH study and bronchoscopy, at the present authors' centre, were very similar to those from the group as a whole.

It must be acknowledged that the mere presence of an abnormality cannot be taken as evidence of causality of the clinical problem. This can probably only be established by double-blind therapeutic trials on an $n=1$ basis or prospective intervention studies in large groups of young children. This report is intended to be descriptive and hypothesis-generating, and to form the basis for the design of intervention studies in this age group. The current authors have documented three broad diagnostic groups: 1) eosinophilic airway inflammation presumed to be asthma, with or without reflux; 2) predominant gastro-oesophageal reflux; and 3) bronchial infection. In a fourth group, no consistent abnormality was found. Clearly, the vindication of the invasive approach of the present study will only come if intervention studies can show a clinical benefit for the child in terms of outcome. To achieve this, studies with protocol-driven treatment of the categories assigned above need to be performed.

In summary, investigations, including bronchoscopy, in preschool children with severe, recurrent wheeze can be performed safely and yield new potentially clinically relevant information, especially with regard to structural airway abnormalities, eosinophilic airway inflammation, bacterial infection and gastro-oesophageal reflux. However, it is essential that these tests, especially bronchoscopy, are performed in a centre with appropriately trained and experienced personnel.

\section{REFERENCES}

1 Kuehni CE, Davis A, Brooke AM, Silverman M. Are all wheezing disorders in very young (preschool) children increasing in prevalence? Lancet 2001; 357: 1821-1825.

2 Stevens CA, Turner D, Kuehni CE, Couriel JM, Silverman M. The economic impact of preschool asthma and wheeze. Eur Respir J 2003 21, 6: 1000-1006.

3 Covar RA, Spahn JD. Treating the wheezing infant. Pediatr Clin North Am 2003; 50: 631-654.

4 Cane RS, Ranganathan SC, McKenzie SA. What do parents of wheezy children understand by "wheeze"? Arch Dis Child 2000; 82: 327-332.

5 Saglani S, Lenney W. Difficult asthma in the pre-school child. Paediatr Respir Rev 2004; 5: 199-206.

6 Bush A. Treatment options of asthma in infancy. Pediatr Pulmonol Suppl 2004; 26: S20-S22.

7 de Jongste JC, Janssens HM, Van der Wouden J. Effectiveness of pharmacotherapy in asthmatic preschool children. Allergy 2002; 57: Suppl. 74, 42-47.

8 Kaditis AG, Gourgoulianis K, Winnie G. Anti-inflammatory treatment for recurrent wheezing in the first five years of life. Pediatr Pulmonol 2003; 35: 241-252.

9 Chan EY, Dundas I, Bridge PD, Healy MJ, McKenzie SA. Skin-prick testing as a diagnostic aid for childhood asthma. Pediatr Pulmonol 2005; 39: 558-562.

10 Bamberg C, Kalache KD. Prenatal diagnosis of fetal growth restriction. Fetal Neonatal Med 2004; 9: 387-394.

11 Guidelines for the performance of the sweat test for the investigation of cystic fibrosis in the UK (2003). Report from the Multi-Disciplinary Working Group. http:// acb.org.uk/docs/sweat.pdf. Date last updated: November 2003; Date last accessed: October 252005.

12 Vandenplas $Y$, Goyvaerts $H$, Helven R, Sacre L. Gastroesophageal reflux, as measured by 24-hour $\mathrm{pH}$ monitoring, in 509 healthy infants screened for risk of 
sudden infant death syndrome. Pediatrics 1991; 88: 834-840.

13 Bush A, Cole P, Hariri M, et al. Primary ciliary dyskinesia: diagnosis and standards of care. Eur Respir J 1998; 12 982-988.

14 Saglani S, Payne DN, Nicholson AG, Scallan M, Haxby E, Bush A. The safety and quality of endobronchial biopsy in children under five years old. Thorax 2003; 58: 1053-1057.

15 Midulla F, de Blic J, Barbato A, et al. Flexible endoscopy of paediatric airways. Eur Respr J 2003; 22: 698-708.

16 Chan EY, McKenzie SA. Normative data for total serum immunoglobulin E measurements in children of three ethnicities. Clin Exp Allergy 2003; 33: 752-756.

17 Weiser MR, Pechet TT, Williams JP, et al. Experimental murine acid aspiration injury is mediated by neutrophils and the alternative complement pathway. J Appl Physiol 1997; 83: 1090-1095.

18 Knauer-Fischer S, Ratjen F. Lipid-laden macrophages in bronchoalveolar lavage fluid as a marker for pulmonary aspiration. Pediatr Pulmonol 1999; 27: 419-422.

19 Kajetanowicz A, Stinson D, Laybolt KS, Resch L. Lipidladen macrophages in the tracheal aspirate of ventilated neonates receiving Intralipid: a pilot study. Pediatr Pulmonol 1999; 28: 101-108.

20 Jain A, Patwari AK, Bajaj P, Kashyap R, Anand VK. Association of gatroesophageal reflux disease in young children with persistent respiratory symptoms. J Trop Pediatr 2002; 48: 39-42.
21 Sheikh S, Stephen T, Howell L, Eid N. Gastroesophageal reflux in infants with wheezing. Pediatr Pulmonol 1999; 28: 181-186.

22 Gold BD. Asthma and gastroesophageal reflux disease in children: exploring the relationship. J Pediatr 2005; 146: Suppl. 3, S13-S20.

23 Hsu JY, Lien HC, Chang CS, Chen GH. Abnormal acid reflux in asthmatic patients in a region with low GERD prevalence. J Gastroenterol 2005; 40: 11-15.

24 Leggett JJ, Johnston BT, Mills M, Gamble J, Heaney LG. Prevalence of gastroesophageal reflux in difficult asthma: relationship to asthma outcome. Chest 2005; 127: 1227-1231.

25 Weinberger M. Gastroesophageal reflux disease is not a significant cause of lung disease in children. Pediatr Pulmonol Suppl 2004; 26: 197-200.

26 Schellhase DE, Fawcett DD, Schutze GE, Lensing SY, Tryka AF. Clinical utility of flexible bronchoscopy and bronchoalveolar lavage in young children with recurrent wheezing. J Pediatr 1998; 132: 312-318.

27 Sim D, Griffiths A, Armstrong D, Clarke C, Rodda C, Freezer N. Adrenal suppression from high-dose inhaled fluticasone propionate in children with asthma. Eur Respir J 2003; 21: 633-636.

28 Grier DG, Halliday HL. Effects of glucocorticoids on fetal and neonatal lung development. Treat Respir Med 2004; 3: 295-304.

29 Saglani S, Malmstrom K, Pelkonen AS, et al. Airway remodeling and inflammation in symptomatic infants with reversible airflow obstruction. Am J Respir Crit Care Med 2005; 26: 197-200. 